The Memorial Thesis of the Best Poster Award (Recommended article)

Recommender: Division of Cellular Signaling, Institute for Advanced Medical Research, Keio University School of Medicine, Prof. Yutaka KAWAKAMI

\title{
Prospects for personalized combination immunotherapy for solid tumors based on adoptive cell therapies and immune checkpoint blockade therapies
}

\author{
Daiki Kato $^{* 1,2}$, Tomonori Yaguchi ${ }^{* 1}$, Takashi IwatA ${ }^{* 1}$, Kenji MoriI ${ }^{* 1}$, \\ Takayuki NaKAGAWA $^{* 2}$, Ryohei NishimURA ${ }^{* 2}$ and Yutaka KaWAKAmi ${ }^{* 1}$ \\ ${ }^{* 1}$ Division of Cellular Signaling, Institute for Advanced Medical Research, Keio University School of Medicine \\ ${ }^{* 2}$ Laboratory of Veterinary Surgery, Graduate School of Agricultural and Life Sciences, The University of Tokyo
}

(Accepted February 1, 2017)

summary

\begin{abstract}
Immune checkpoint blockade (ICB) and adoptive cell therapies (ACT) with antigen-receptor gene-engineered T cells have been shown to be successful for a limited number of patients with solid tumors. Responders to ICB therapy typically have T cell-inflamed tumors. Thus, it is important to develop strategies that convert non-T cell-inflamed tumors to $\mathrm{T}$ cell-inflamed tumors. Although chimeric antigen receptor transduced T (CAR-T) cell therapy targeting hematological malignancies demonstrated durable clinical responses, the success of gene-engineered $\mathrm{T}$ cell therapies in solid tumors is hampered by a lack of unique antigens, antigen loss in cancer cells, and the immune-suppressive tumor microenvironment (TME) of solid tumors. However, gene-engineered $\mathrm{T}$ cells possess strong killing activity and cytokine production capacity, which can induce antigen spreading and modulate the TME of non-T cell-inflamed tumors seen in non-responders to ICB therapy. Immune responses against cancer are highly heterogeneous, not only between tumor types, but also within a patient or between different patients with the same type of cancer, indicating that personalized immunotherapy should be employed, based on the immune status of the individual patient. Here, we offer our perspective for personalized combination immunotherapy for solid tumors based on ACT and ICB therapies.
\end{abstract}

Key words__ tumor immunology; tumor microenvironment; immune checkpoint blockade therapy; adoptive cell therapy; chimeric antigen receptor transduced $\mathrm{T}(\mathrm{CAR}-\mathrm{T})$ cell therapy

\section{Immune checkpoint blockade therapies}

Cytotoxic $\mathrm{T}$ cells can specifically recognize tumor antigen (tumor $\mathrm{Ag}$ ) and eliminate cancers $^{1-4)}$. As demonstrated by recent evidence from study of immune checkpoint blockade (ICB) and adoptive T cell therapies, an entirely immunologic approach can treat a wide range of human cancers. ICB therapies, which use monoclonal antibodies (mAbs) that target inhibitory immune checkpoint molecules such as cytotoxic $\mathrm{T}$ lymphocyteassociated protein $4(\text { CTLA- } 4)^{5)}$ and programmed cell death protein 1 (PD-1)/programmed cell death-ligand protein $1(\mathrm{PD}-\mathrm{L} 1)^{6}$, , have demonstrated durable clinical responses in various cancers, including melanoma ${ }^{7,8)}$, lung cancer ${ }^{8,9)}$, renal cell carcinoma ${ }^{8,10)}$, bladder cancer ${ }^{11)}$, ovarian cancer ${ }^{12)}$, triple-negative breast cancer ${ }^{13)}$, gastric cancer $^{14)}$, Hodgkin's lymphoma ${ }^{15)}$, and gastrointestinal and endometrial cancers associated with defects in DNA mismatch repair ${ }^{16)}$. In melanoma, lung cancer, and renal cell carcinoma, clinical response rates as high as $20-30 \%$ have been reported ${ }^{8,9)}$. The observed anti-tumor responses were associated with the activation and expansion of tumor Ag-specific $\mathrm{T}$ cells ${ }^{17}{ }^{18)}$, and responding patients showed high pre-treatment tumor infiltration of $\mathrm{T}$ cells compared to non-responding patients ${ }^{19)}$. This evidence suggests that conversion of non-T cell-inflamed tumors to $\mathrm{T}$ cell-inflamed tumors would improve response to ICB therapies.

\section{Adoptive cell therapies}

$\mathrm{T}$ cells are considered the final effectors of immune-mediated cancer regression, hence direct administration of tumor Ag-specific T cells has been developed into adoptive $\mathrm{T}$ cell therapies $(\mathrm{ACT})^{20)}$ (Fig. 1). To prepare $\mathrm{T}$ cells for ACT, tumor-infiltrating lymphocytes (TILs) are harvested from surgically removed tumor metastases and expanded ex vivo. Administration of TILs demonstrated durable clinical responses in patients 
with melanoma and cervical cancer $^{21,22)}$. TIL therapy for patients with advanced melanoma showed a 52-72\% objective response rate based on the Response Evaluation Criteria in Solid Tumors (RECIST), and 19 of the 93 patients (20\%) achieved complete tumor regression beyond 3 years $^{22)}$. To broaden the efficacy of ACT, the antigens recognized by TILs have been studied extensively. A recent report has demonstrated that mutated antigens in a non-melanoma epithelial cancer (i.e. gastrointestinal cancer) can give rise to immune responses, despite the low number of nonsynonymous somatic mutations in these cancers ${ }^{3)}$. While TILs can be grown in vitro from virtually all tumors, TILs that demonstrate a clinical response have only been produced from melanomas ${ }^{20)}$.

\section{Gene-engineered cell therapies}

Progress in genetic engineering technologies has enabled the efficient generation of antigen-receptor geneengineered $\mathrm{T}$ cells, including $\mathrm{T}$ cell receptor transduced $\mathrm{T}$ (TCR-T) cells and chimeric antigen receptor transduced $\mathrm{T}$ (CAR-T) cells (Fig. 1). Use of gene-engineered T cells obviates the requirement for surgery because these T cells can be isolated from blood samples, and viral or non-viral transduction of receptors confers tumor Ag specificity ${ }^{23)}$. As the same antigen receptor can be used for a large population of cancer patients and gene-engineered $\mathrm{T}$ cells can potentially be produced on a large scale, geneengineered $\mathrm{T}$ cells may be useful as an off-the-shelf formulation. Recent experience with sipuleucel-T (trade name Provenge), a gene-engineered cell product for prostate cancer, demonstrated the feasibility of processing patient immune cells in a central manufacturing facility and returning them to the patient in a manner that has gained US Food and Drug Administration (FDA) regulatory approval $^{24)}$.

\section{TCR-T cell therapies}

TCR-T cells are made by transduction of a cloned T cell receptor (TCR) that recognizes tumor Ag presented within the groove of a specific major histocompatibility complex (MHC). The engineered TCR triggers T cell activation via the signal transduction machinery employed by endogenous $\mathrm{TCR}^{25)}$. Several reports have been published regarding clinical trials of TCR-T cell therapy. In an early clinical trial, 17 patients with advanced melano- ma displaying human leukocyte antigen (HLA)-A*0201 were treated with TCR-T cells specific for a HLA-A2restricted epitope derived from melanocyte-differentiating antigen (MART-1). The study resulted in long-term persistence of the infused cells and accompanying tumor regression in 2 of 17 patients, without toxicities attributed to the transferred cells ${ }^{26}$. A subsequent study with an affinity-enhanced MART-1-specific TCR reported tumor regression in 6 of 20 patients $(30 \%)$. Some patients showed moderate but non-lethal toxicity in tissues where melanocytes are present, such as the skin, eyes, and ears $^{27)}$. A recent study using modified high-avidity TCR targeting a HLA-A2-restricted epitope derived from a cancer germline antigen (NY-ESO-1) demonstrated a good response in 11 of 20 patients $(55 \%)$ with advanced melanoma (4 CRs, 3 of these CRs were ongoing after $>36$ months) and 11 of 18 (61\%) patients with synovial cell sarcoma, a non-melanoma solid tumor (1CR). No toxicities attributed to the transferred cells were record$\mathrm{ed}^{28,29)}$. However, in a study using affinity-enhanced TCR against HLA-A*01-restricted MAGE-A3, 2 patients with melanoma and myeloma developed cardiogenic shock and died within a few days of T-cell infusion. This adverse effect was caused by recognition of an unrelated peptide derived from cardiomyocytes by the affinityenhanced $\mathrm{TCR}^{30,31)}$

\section{CAR-T cell therapies}

TILs are difficult to generate in non-melanoma cancers, and TCR-T cells recognize only specific MHC alleles, limiting their use to a restricted subset of patients and making them vulnerable to $\mathrm{MHC}$ downregulation and immune escape ${ }^{32)}$. To address these limitations, a new concept of gene-engineered $\mathrm{T}$ cells has been developed. A versatile class of receptors known as chimeric antigen receptors (CARs) are generated by combining the antigen-binding domain of a single-chain variable fragment $(\mathrm{scFv})$ from a $\mathrm{mAb}$ that recognizes tumor $\mathrm{Ag}$ with intracellular signaling motifs that are capable of $\mathrm{T}$ cell activation ${ }^{33)}$. Because their ability to recognize antigens is enabled by antibody binding, CARs can mimic endogenous TCR-mediated activation without the drawbacks of classical MHC restriction. Moreover, although endogenous TCRs are restricted by selection in the thymus, antibody-redirected CARs can accommodate 


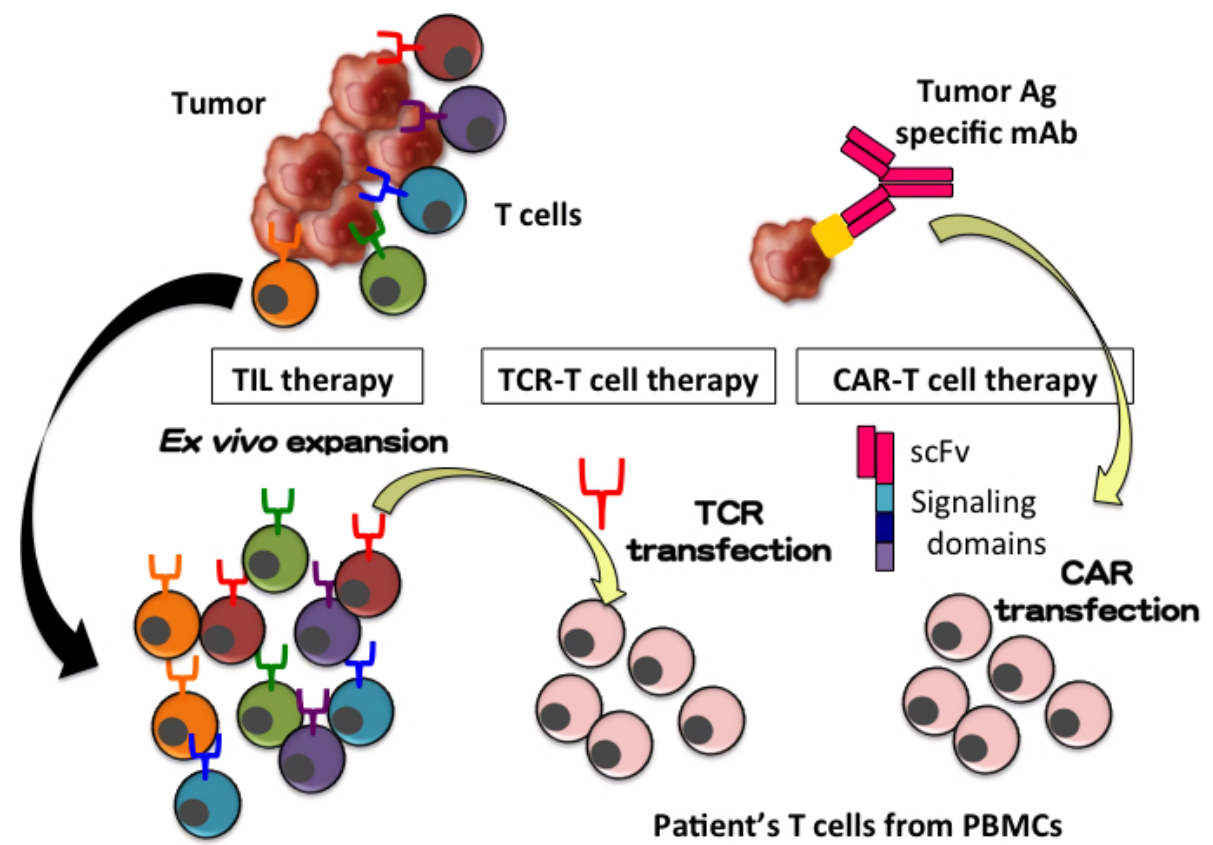

Fig. 1. Summary of concepts of adoptive T cell therapies (ACT)

Three types of tumor Ag-specific T cells are used for ACT. For tumor-infiltrating lymphocyte (TIL) therapy, TILs are harvested from surgically removed tumor tissue and expanded ex vivo. Because TILs recognize a variety of tumor antigens, TIL therapy is a polyclonal antigentargeting therapy. For T cell receptor transduced T (TCR-T) cell therapy, tumor Ag-specific TCR sequences are obtained from tumor Agspecific T cells. Monoclonal antigen-targeting TCR-T cells are generated by transfection of these tumor Ag-specific TCR genes in patient T cells derived from PBMCs. For chimeric antigen receptor transduced T (CAR-T) cell therapy, CARs are generated by connecting intracellular signaling domains and a single-chain variable fragment $(\mathrm{scFv})$ from a mAb that recognizes tumor Ag. The CAR genes can also be transfected into $\mathrm{T}$ cells from patient-derived PBMCs.

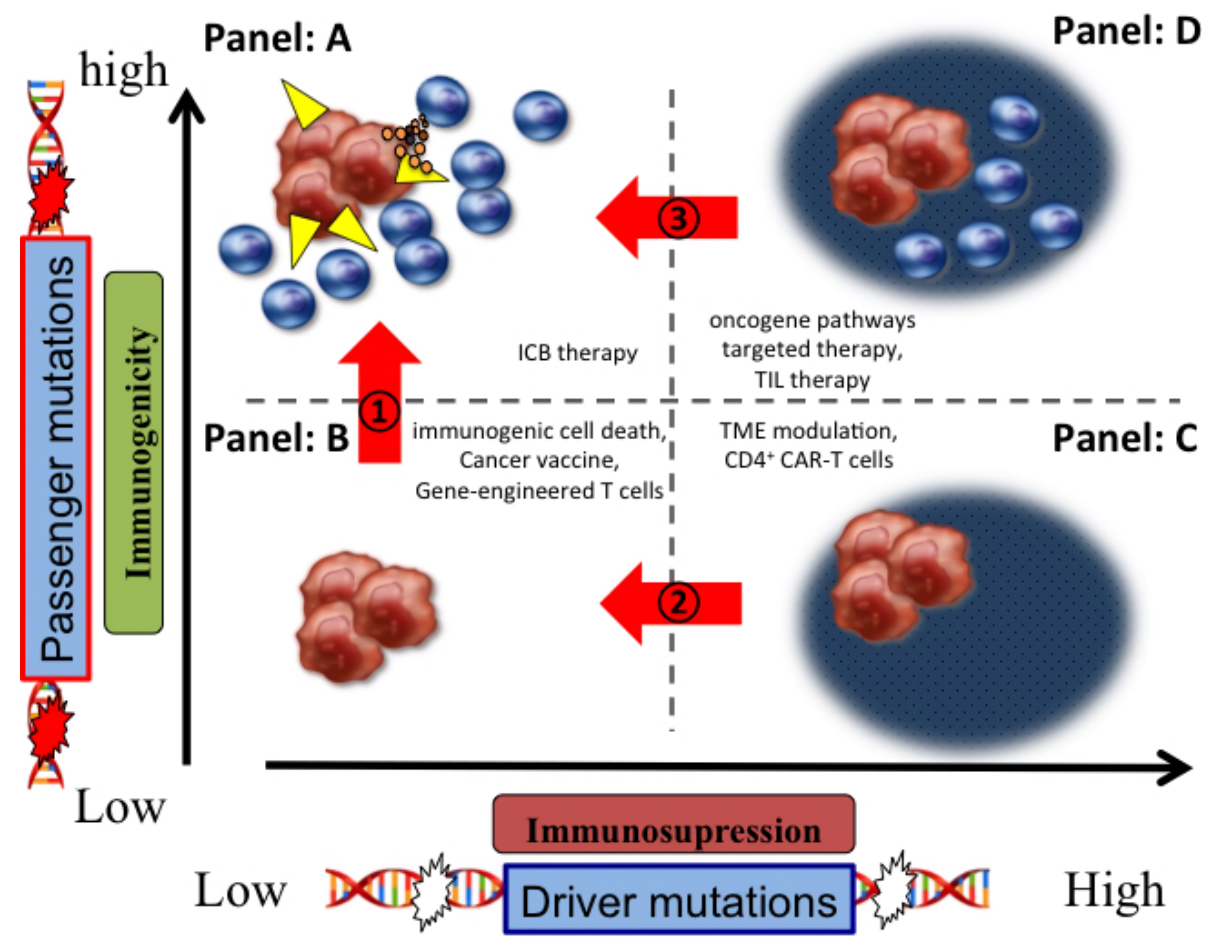

Fig. 2. Prospects for combination adoptive immunotherapies

The immune status of tumors can be categorized into four types, based on mutation-derived immunogenicity, mainly passenger mutations, and mutation-derived immunosupression, mainly driver mutations. The tumor shown in panel A possesses a large amount of imunogenic mutations; hence, tumor Ag-specific T cells have infiltrated strongly and immune checkpoint blockade (ICB) therapies would be effective. In contrast, the tumor shown in panels $\mathrm{C}$ and $\mathrm{D}$ possesses a highly immunosupressive tumor microenvironment (TME), hence modulation of the TME would be needed. Because the tumor in panel B shows very weak immunognicity, treatment for enhancement of immune recognition (e.g., immunogenic cell death) would be needed. Because patient immune status is highly heterogeneous, personalized combination immunotherapy should be considered. 
infinite antigenic diversity and operate at affinities in the nanomolar range $\mathrm{e}^{34,35)}$. As an additional advantage, the signaling domain of CAR constructs can be modulated to exhibit various $\mathrm{T}$ cell functions, including expansion, survival, cytokine secretion, tissue-selectivity, and safety $^{36-40)}$.

\section{Clinical trials of CAR-T cell therapies}

Many clinical trials of CD19-specific CAR-T cell therapies targeting $\mathrm{B}$ cell malignancies demonstrated objective regression of cancer in patients with acute lymphoblastic leukemia (ALL), chronic lymphocytic leukemia (CLL), and various other types of B cell lymphoma. The first successful application of CD19specific CAR-T cell therapy was observed in patients with follicular lymphoma ${ }^{41)}$, and the treatment target was subsequently extended to other chemotherapy-refractory B cell cancers ${ }^{42,43}$. For example, CAR-T cell therapy of pediatric and adult patients with ALL demonstrated a complete remission rate of approximately $90 \%$, with sustained remission for up to 2 years ${ }^{44)} ; 57 \%$ of patients with CLL showed an objective response, including complete remission $(29 \%)^{45)}$. Because normal B cells express CD19 antigen, B cell aplasia and deficiencies in circulating immunoglobulins (Igs) are frequently observed in patients treated with CD19-specific CAR-T cell therapies. These toxicities can be managed with infusion of Igs. Many patients also exhibit cytokine release syndrome after $\mathrm{T}$ cell infusion. In most cases, these side effects can be managed with aggressive supportive care or immunosuppression including steroids and cytokine-specific antibodies $^{46)}$. Because CD19-specific CAR-T cell therapy has shown a favorable and lasting clinical outcome compared with conventional radio- or chemotherapy, CD19-specific CAR-T cell therapy may enter mainstream care for many B cell malignancies in the next a few years and the FDA has recognized it as a "breakthrough therapy". Although CAR-T cell therapies are effective in treating hematological malignancy, use of CAR-T cell therapies for solid tumors has encountered many difficulties, including limitation of tumor-specific antigens expressed on the surface of solid tumors and the need for $\mathrm{T}$ cell infiltration into tumor tissue where the environment is heavily immunosuppressive. A trial with GD2-specific CAR-T cells resulted in tumor regression in 3 of 11 patients with neuroblastoma, including 2 sustained CRs, without severe adverse effects ${ }^{47)}$. In addition, a study of CD171-specific CAR-T cells demonstrated a partial response in 1 of 6 patients with neuroblastoma ${ }^{48)}$. Because shared tumor Ags are occasionally expressed in normal tissues and recognized by CAR-T cells, substantial unexpected on-target off-tumor toxicities have occurred. For example, a trial of CAR-T cells targeting carbonic anhydrase IX (CAIX), overexpressed in renal cell carcinoma, caused severe liver dysfunction due to cholangitis, without cancer regression. The expression of CAIX in epithelial cells of the bile duct results in on-target toxicities ${ }^{34)}$. In addition, a lethal adverse effect was reported in a study of ERBB2-specific CAR-T cells expressing $\mathrm{scFv}$ from the humanized $\mathrm{mAb}$ trastuzumab, resulting in fatal respiratory failure, with inflammatory cytokine release in the lung within 15 minutes after intravenous infusion of $10^{10}$ cells $^{49)}$. It was speculated that many of the administered $\mathrm{T}$ cells localized in the lungs and were activated by recognition of ERBB2 on lung epithelial cells, leading to extreme cytokine release. In contrast, a recent trial employing infusion of $10^{8}$ cells of ERBB2-specific CAR-T cells using a different antiERBB2 Ab clone (FRP5, a mouse anti-human ERBB2 $\mathrm{mAb}$ ) demonstrated no significant toxicities, although no significant tumor reduction was observed in this $\operatorname{trial}^{50)}$. These results from clinical trials might indicate the potential of ERBB2 as a therapeutic antigen and the importance of careful selection of the Ab clones used for production of CAR-T cells.

Clinical trials of ACT with gene-engineered T cells, such as NY-ESO-1 TCR-T cells and GD2-specific CAR-T cells, have shown that gene-engineered $\mathrm{T}$ cells have the potential to show anti-tumor effects without damaging normal tissue in patients with solid tumors. However, on-target off-tumor toxicity of gene-engineered $\mathrm{T}$ cell therapies causes serious adverse effects, which have been reported for TCRs with mouse-derived gp100, mousederived CEA, affinity-enhanced MART-1, affinityenhanced MAGE-A3, and CARs directed against ERBB2 and $\mathrm{CAIX}^{27,31,49,51)}$. The most important factor is the selection of the target antigen; the most desirable antigen must be expressed only on tumor cells and not on normal tissue. However, even if the target antigen is expressed in normal cells, it can be a possible therapeutic target. For 
example, no severe adverse effects have been reported for mesothelin-specific CAR-T cell therapies, even though a substantial amount of mesothelin is expressed in normal mesothelial cells; some objective responses to these therapies have been documented. There might be an unknown mechanism for the specific recognition of the antigen expressed on cancer cells by CAT-T cells ${ }^{52)}$. This case might lead to relaxation of the restriction of target antigens for gene-engineered $\mathrm{T}$ cell therapies.

\section{Immunosuppressive TME and ACT therapies}

The immunosuppressive status of the tumor microenvironment (TME) is a major problem in cancer immunotherapies, as it reduces the anti-tumor effects of immunotherapies. Two types of immunosuppression mechanisms operate in the $\mathrm{TME}^{53,54)}$. One is tumorintrinsic immunosuppression, which is induced by genetic alterations related to cancer. The other is local adaptive immunosuppression, which is triggered by tumor-specific $\mathrm{T}$ cells infiltrated in tumors. The former operates via multiple mechanisms that employ various immunosuppressive molecules triggered by genetic alterations, including activation of various oncogene pathways such as the Wnt- $\beta$-catenin signaling pathway ${ }^{55,56}$, the MAPK signaling pathway ${ }^{57,58)}$, the JAK-STAT3 signaling pathway ${ }^{57,59)}$, and the NF- $\mathrm{B}$ signaling pathway ${ }^{60)}$. The activation of tumor-intrinsic oncogene pathways induces tumor cells to produce suppressive molecules such as cytokines and chemokines, mediating exclusion of $\mathrm{T}$ cells from the TME. Small molecule inhibitors, such as $\beta$-catenin and BRAF inhibitors, can modulate the activated oncogene pathways $^{60-62)}$. Based on current knowledge, it is conceivable that combination therapies of ACT with inhibition of the activated oncogene pathways using small molecule inhibitors could work synergistically to exhibit anti-tumor effects through mechanisms that restore T-cell infiltration. In a recent study, BRAF inhibitors improved the anti-tumor activity of ACT in mouse models ${ }^{62)}$.

The latter mechanism, local adaptive immunosuppression, is triggered by tumor-specific cytotoxic lymphocytes (CTLs), whereby cytokines, such as IFN- $\gamma$ produced by anti-tumor $\mathrm{T}$ cells, induce immunecheckpoint molecules such as PD-L1, immunosuppressive indoleamine (IDO), which catabolizes tryptophan, or induce chemokines. We and others reported the potential effectiveness of combination therapies of ACT with ICB therapies targeting molecules such as PD-1, TIGIT, LAG3, and Tim3 that are expressed on tumor Ag-specific $\mathrm{T}_{\text {cells }}{ }^{63-65)}$. In ACT therapies, adaptive immunosuppression can theoretically occur, and it is also conceivable that combination therapies of ACT with ICB therapies could be effective through restoration of T-cell function.

\section{Modulation of the TME by gene-engineered $T$ cell therapies}

Gene-engineered $\mathrm{T}$ cell therapies, which typically target monoclonal antigens, have a disadvantage compared to TIL therapies, which use polyclonal TILs. This disadvantage was exemplified by a CD19-specific CAR-T cell therapy, which led to the loss of CD19 expression in leukemia cells ${ }^{44)}$. Although it might be not easy to cure bulky tumors using only gene-engineered $\mathrm{T}$ cell therapies, their strong killing and cytokine production abilities could be employed for tumor Ag releasing and modulation of the immunosuppressive TME. The evidence for contribution of antigen spreading to favorable outcomes in CAR-T cell therapy was suggested in an immunocompetent mouse model. This study showed that the cured mice which were treated with ACT of murine EGFRvIII-specific CAR-T cell therapy did not develop tumors upon rechallenge with EGFRvIII negative matched tumor ${ }^{66)}$. This study suggests that the strong anti-tumor abilities of gene-engineered $\mathrm{T}$ cell therapies could potentially trigger anti-tumor immune reaction and convert the non-T cell-inflamed TME seen in ICB non-responders to the T cell-inflamed TME.

\section{$\mathrm{CD4}^{+}$CAR-T cell therapies}

Because $\mathrm{CD}^{+} \mathrm{T}$ cells have the ability to directly target, engage, and destroy tumor cells, current basic and clinical research on CAR-T cells focuses mainly on improving $\mathrm{CD}^{+}$CAR-T cell-mediated cellular immunotherapy. Because most tumors do not express MHC class II molecules, $\mathrm{CD}^{+} \mathrm{T}$ cells cannot recognize tumor cells. However, $\mathrm{CD}^{+} \mathrm{T}$ cells transduced with CAR can potentially recognize target $\mathrm{Ag}$ and produce various cytokines. Modulation of the immunosuppressive TME has been reported to attract anti-tumor $\mathrm{T}$ cells to the tumor site by production of Th cytokines from transferred $\mathrm{CD} 4^{+}$ $\mathrm{T}$ cells. Moreover, there have been many evidence that 
tumor infiltration and interaction of $\mathrm{CD}^{+} \mathrm{T}$ cells with immune cells at the tumor site are associated with a good $\operatorname{prognosis}^{67)}$.

Th1 and Th2 cells are the best-characterized subtypes of helper T cells. Th1 cells are characterized by production of cytokines such as IFN- $\gamma$, TNF- $\alpha$, monocyte chemotactic protein-1 (MCP-1), and macrophage inflammatory protein-1 $\alpha$ (MIP1 $\alpha)$, and are thought to be primarily responsible for immune responses against intracellular pathogens either by enhancing the $\mathrm{CD}^{+} \mathrm{T}$ cell response or by directly activating macrophages to phagocytose intracellular pathogens. Th2 cells produce interleukin IL-4, -5 , and -10 , and mainly respond to extracellular pathogens by orchestrating humoral immunity and promoting allergic inflammatory responses. Although IFN- $\gamma$ secreting Th1 cells may be more potent, both of these subtypes have been reported to demonstrate anti-tumor function $^{68,69)}$. The major anti-tumor effects of Th1 cells may result from the secretion of large amounts of IFN- $\gamma$, as well as chemokines that enhance priming and expansion of $\mathrm{CD}^{+} \mathrm{T}$ cells. Th1 cells also help to recruit natural killer (NK) cells and type I macrophages to tumor sites, and act in concert for tumor eradication ${ }^{70-72)}$. Clinical data have also demonstrated the anti-tumor effects of Th1-phenotype $\mathrm{CD}^{+} \mathrm{T}$ cell adoptive transfer. Tran et al. showed the anti-tumor effect of IFN- $\gamma$-producing $\mathrm{CD}^{+}$ TIL therapy that is specific to tumor-mutational $\mathrm{Ag}$ in a patient with cholangiocarcinoma ${ }^{73)}$. Hunder et al. reported a durable response in a patient with advanced melanoma treated with in vitro generated autologous IFN- $\gamma$-producing $\mathrm{CD}^{+} \mathrm{T}$ cell clones that recognize the melanoma-associated antigen NY-ESO-1 ${ }^{74)}$. This patient experienced a complete response and has remained disease-free for 2 years, with persistence of the transferred cells. In addition, induction of endogenous $\mathrm{CD} 8^{+} \mathrm{T}$ cells against various melanoma antigens other than NYESO-1, such as MAGE-3 and MART-1, was detected. Although this result was only published as a case report, it suggests that Th1 cells have the potential to induce antigen spreading and tumor Ag-specific $\mathrm{CD}^{+} \mathrm{T}$ cells.

Other Th subsets have also been demonstrated to show anti-tumor effects. Th17 are characterized primarily by production of the cytokines IL-17A and IL-17F ${ }^{75)}$. IL-17A in turn induces the expression of multiple chemokines, such as CCL2, CCL7, CXCL1, and CCL20, that promote inflammatory responses. Although the influence of Th17 cells and IL-17A has been reported for multiple patients with cancer, their contribution to tumor progression and immune protection remains unclear $^{76}$. Antigen-specific Th17 cells differentiated in vitro showed antitumor activity against murine B16 melanoma stronger than that of Th1 cells ${ }^{77,78)}$. These Th17 cells promoted production of CCL20, which led to recruitment of dendritic cells and effector $\mathrm{T}$ cells into the tumor site, as well as the induction of tumor Ag-specific T cells.

A pioneering study revealed the ability of a new subset of Th9 cells for control of tumor growth ${ }^{79)}$. In a B16 melanoma model, Purwar et al. showed that the antitumor effects of adoptive transfer of antigen-specific Th9 cells were greater than those of transferred Th1 or Th17 cells. Another report demonstrated the anti-tumor mechanism of Th9 cells, which induced strong endogenous tumor $\mathrm{Ag}$-specific $\mathrm{CD}^{+} \mathrm{T}$ cell responses by promoting $\mathrm{Ccl} 20 /$ Ccr6-dependent recruitment of DCs to the tumor site, combined with $\mathrm{CD}^{+} \mathrm{T}$ cell priming ${ }^{80)}$. In addition, IL21 produced by Th9 and Th17 cells is a well-known stimulator of IFN- $\gamma$ production, and it enhances the cytolytic activity of NK cells and $\mathrm{CD}^{+} \mathrm{T}$ cells. IL-21 enhances generation of human tumor Ag-specific $\mathrm{CD}^{+} \mathrm{T}$ cells and promotes the effector functions of generated Ag-specific $\mathrm{CTLs}^{81)}$.

Together, these data provide a strong impetus to investigate the anti-tumor efficacy of adoptive transfer of $\mathrm{CD}^{+}$Th cells. Adoptive transferred $\mathrm{CD}^{+}$Th cells possess the potential for TME modulation, enhancement of effector $\mathrm{T}$ cell function, and induction of Ag-specific endogenous effector $\mathrm{T}$ cells against patient-specific abundant neo-Ags.

\section{Conclusion}

Recent progress in cancer immunotherapy has provided direction for the future improvement of cancer immunotherapy. Particularly, ICB therapies and T cell-based ACT showed robust clinical responses; however, these therapies have only been effective in a limited number of patients. Clinical responses to ICB were correlated with the non-synonymous mutation burden, which can potentially generate immunogenic neo-Ags ${ }^{17,18)}$, and preexisting effector $\mathrm{T}$ cell infiltration ${ }^{19)}$. Notably, immune infiltrates are highly heterogeneous, not only between 
tumor types, but also within a patient or between different patients with the same cancer type, indicating that personalized immunotherapy should be employed based on the immune status of the individual patient. Thus, we propose classification of tumors into four groups, on the basis of their immunogenicity and immune suppression in the $\mathrm{TME}^{82)}$ (Fig. 2). Patients with sufficient pre-existing anti-tumor $\mathrm{T}$ cells and local adoptive immune suppression, including PD-1-related suppression (shown in Panel A), will show a better response to ICB therapies, such as anti-PD-1 Ab therapies. In patients with few pre-existing anti-tumor $\mathrm{T}$ cells, due to low tumor immunogenicity (shown in Panel B), an immune reaction needs to be induced. Because these patients need immunogenic tumor $\mathrm{Ag}$ release, treatment with immunogenic cell death, cancer vaccine therapies, or ACT with gene-engineered $\mathrm{T}$ cells ${ }^{52)}$ would convert the TME from B to A (arrow 1). In patients with few pre-existing anti-tumor $\mathrm{T}$ cells and strong tumor-intrinsic immune suppression (shown in Panel C), the immunosuppressive TME needs to be modulated. Because various mechanisms of immune suppression may be operating in the TME of these patients, oncogene pathway-targeted therapies, TME cytokine modulation by ACT of $\mathrm{CD}^{+} \mathrm{CAR}-\mathrm{T}$ cells ${ }^{74)}$, or other immune suppression blockades could be used to convert the TME from $\mathrm{C}$ to $\mathrm{B}$ (arrow 2). In patients with moderate pre-existing anti-tumor $\mathrm{T}$ cells, because of tumor immunogenicity and strong tumor-intrinsic immune suppression (shown in Panel D), the immunosuppressive TME needs to be modulated. In these patients, oncogene pathway-targeted therapies, other immune suppression blockades, or TIL therapies could be used to convert TME from D to A (arrow 3). Overall, personalized combination immunotherapy should be considered for improvement of cancer immunotherapy.

\section{Acknowledgements}

This work was supported by Grants-in-aid for Scientific Research (26221005 and 15K09783) from the Ministry of Education, Culture, Sports, Science and Technology (MEXT) of Japan, and the Project for Cancer Research And Therapeutic Evolution (P-CREATE) (16cm0106305 h0001) from Japan Agency for Medical Research and Development (AMED). We thank Mr. JeongHoon Park for preparation of the manuscript.

\section{References}

1) Kawakami, Y., et al.: Cloning of the gene coding for a shared human melanoma antigen recognized by autologous T cells infiltrating into tumor. Immunology. 91: 3515-3519, 1994.

2) Kawakami, Y., et al.: Recognition of shared melanoma antigens in association with major HLA-A alleles by tumor infiltrating $\mathrm{T}$ lymphocytes from 123 patients with melanoma. J Immunother. 23: 17-27, 2000.

3) Tran, E., et al.: Immunogenicity of somatic mutations in human gastrointestinal cancers. Science. 350: 1387-1390, 2015.

4) Galon, J., et al.: Validation of the Immunoscore (IM) as a prognostic marker in stage I/II/III colon cancer: Results of a worldwide consortium-based analysis of 1,336 patients. J Clin Oncol. 34 (Suppl.): abstr 3500, 2016.

5) Mokyr, M.B., et al.: Realization of the therapeutic potential of CTLA-4 blockade in low-dose chemotherapy-treated tumor-bearing mice. Cancer Res. 58: 5301-5304, 1998.

6) Iwai, Y., et al.: Involvement of PD-L1 on tumor cells in the escape from host immune system and tumor immunotherapy by PD-L1 blockade. Proc Natl Acad Sci U S A. 99: 12293-12297, 2002.

7) Hodi, F.S., et al.: Improved Survival with Ipilimumab in Patients with Metastatic Melanoma. $N$ Engl J Med. 363: 711-723, 2010.

8) Topalian, S.L., et al.: Safety, Activity, and Immune Correlates of Anti-PD-1 Antibody in Cancer. $N$ Engl J Med. 366: 2443-2454, 2012.

9) Brahmer, J., et al.: Nivolumab versus Docetaxel in Advanced Squamous-Cell Non-Small-Cell Lung Cancer. N Engl J Med. 373: 123-135, 2015.

10) Motzer, R.J., et al.: Nivolumab versus Everolimus in Advanced Renal-Cell Carcinoma. $N$ Engl J Med. 373: 1803-1813, 2015.

11) Powles, T., et al.: MPDL3280A (anti-PD-L1) treatment leads to clinical activity in metastatic bladder cancer. Nature. 515: 558-562, 2014.

12) Hamanishi, J., et al.: Safety and Antitumor Activity of Anti-PD-1 Antibody, Nivolumab, in Patients With Platinum-Resistant Ovarian Cancer. J Clin Oncol. 33: 4015-4022, 2015.

13) Gibson, J.: Anti-PD-L1 for metastatic triple-negative breast cancer. Lancet Oncol. 16: e264, 2015.

14) Muro, K., et al.: Pembrolizumab for patients with PD-L1-positive advanced gastric cancer (KEYNOTE-012): a multicentre, open-label, phase $1 \mathrm{~b}$ 
trial. Lancet Oncol. 17: 717-726, 2016.

15) Ansell, S.M., et al.: PD-1 Blockade with Nivolum$\mathrm{ab}$ in Relapsed or Refractory Hodgkin's Lymphoma. N Engl J Med. 372: 311-319, 2015.

16) Le, D.T., et al.: PD-1 Blockade in Tumors with Mismatch-Repair Deficiency. $N$ Engl J Med. 372: 2509-2520, 2015.

17) Rizvi, N.A., et al.: Cancer Immunology. Mutational landscape determines sensitivity to PD-1 blockade in non-small cell lung cancer. Science. 348: 124$128,2015$.

18) Snyder, A., et al.: Genetic Basis for Clinical Response to CTLA-4 Blockade in Melanoma. $N$ Engl JMed. 371: 2189-2199, 2014.

19) Tumeh, P.C., et al.: PD-1 blockade induces responses by inhibiting adaptive immune resistance. Nature. 515: 568-571, 2014.

20) Rosenberg, S.A., Restifo, N.P.: Adoptive cell transfer as personalized immunotherapy for human cancer. Science. 348: 62-68, 2015.

21) Stevanović, S., et al.: Complete regression of metastatic cervical cancer after treatment with human papillomavirus-targeted tumor-infiltrating $\mathrm{T}$ cells. J Clin Oncol. 33: 1543-1550, 2015.

22) Rosenberg, S.A., et al.: Durable complete responses in heavily pretreated patients with metastatic melanoma using T-cell transfer immunotherapy. Clin. Cancer Res. 17: 4550-4557, 2011.

23) Singh, H., et al.: Manufacture of $T$ cells using the Sleeping Beauty system to enforce expression of a CD19-specific chimeric antigen receptor. Cancer Gene Ther. 22: 95-100, 2015.

24) Kantoff, P.W., et al.: Sipuleucel-T immunotherapy for castration-resistant prostate cancer. $N$ Engl $J$ Med. 363: 411-422, 2010.

25) Ahmadi, M., et al.: CD3 limits the efficacy of TCR gene therapy in vivo. Blood. 118: 3528-3537, 2011.

26) Morgan, R.A., et al.: Cancer Regression in Patients After Transfer of Genetically Engineered Lymphocytes. Science. 314: 126-129, 2006.

27) Johnson, L.A., et al.: Gene therapy with human and mouse T-cell receptors mediates cancer regression and targets normal tissues expressing cognate antigen. Blood. 114: 535-546, 2009.

28) Robbins, P.F., et al.: A pilot trial using lymphocytes genetically engineered with an NY-ESO-1-reactive T-cell receptor: long-term follow-up and correlates with response. Clin Cancer Res. 21: 1019-1027, 2015 .
29) Robbins, P.F., et al.: Tumor regression in patients with metastatic synovial cell sarcoma and melanoma using genetically engineered lymphocytes reactive with NY-ESO-1. J Clin Oncol. 29: 917-924, 2011.

30) Cameron, B.J., et al.: Identification of a Titinderived HLA-A1-presented peptide as a crossreactive target for engineered MAGE A3-directed T cells. Sci Transl Med. 5: 197ra103, 2013.

31) Linette, G.P., et al.: Cardiovascular toxicity and titin cross-reactivity of affinity-enhanced $\mathrm{T}$ cells in myeloma and melanoma. Blood. 122: 863-871, 2013.

32) Rosenberg, S.A., et al.: Cell transfer therapy for cancer: lessons from sequential treatments of a patient with metastatic melanoma. J Immunother. 26: 385-393, 2003.

33) Gross, G., et al.: Expression of immunoglobulinT-cell receptor chimeric molecules as functional receptors with antibody-type specificity. Proc Natl Acad Sci U S A. 86: 10024-10028, 1989.

34) Lamers, C.H.J., et al.: Treatment of metastatic renal cell carcinoma with autologous T-lymphocytes genetically retargeted against carbonic anhydrase IX: first clinical experience. J Clin Oncol. 24: e20-22, 2006.

35) Hollyman, D., et al.: Manufacturing validation of biologically functional $\mathrm{T}$ cells targeted to CD19 antigen for autologous adoptive cell therapy. $J$ Immunother. 32: 169-180, 2009.

36) Morsut, L., et al.: Engineering Customized Cell Sensing and Response Behaviors Using Synthetic Notch Receptors. Cell. 164: 780-791, 2016.

37) Wu, C.-Y., et al.: Remote control of therapeutic $\mathrm{T}$ cells through a small molecule-gated chimeric receptor. Science. 350: aab4077-aab4077, 2015.

38) Zhong, X.-S., et al.: Chimeric antigen receptors combining 4-1BB and CD28 signaling domains augment PI3kinase/AKT/Bcl-XL activation and CD8+ T cell-mediated tumor eradication. Mol Ther. 18: 413-420, 2010.

39) Zhao, Y., et al.: A herceptin-based chimeric antigen receptor with modified signaling domains leads to enhanced survival of transduced $\mathrm{T}$ lymphocytes and antitumor activity. J Immunol. 183: 55635574, 2009.

40) Milone, M.C., et al.: Chimeric Receptors Containing CD137 Signal Transduction Domains Mediate Enhanced Survival of T Cells and Increased Antileukemic Efficacy In Vivo. Mol Ther. 17: 14531464, 2009. 
41) Kochenderfer, J.N., et al.: Eradication of B-lineage cells and regression of lymphoma in a patient treated with autologous $\mathrm{T}$ cells genetically engineered to recognize CD19. Blood. 116: 4099-4102, 2010.

42) Porter, D.L., et al.: Chimeric Antigen ReceptorModified T Cells in Chronic Lymphoid Leukemia. N Engl J Med. 365: 725-733, 2011.

43) Grupp, S.A., et al.: Chimeric Antigen ReceptorModified T Cells for Acute Lymphoid Leukemia. N Engl J Med. 368: 1509-1518, 2013.

44) Maude, S.L., et al.: Chimeric Antigen Receptor T Cells for Sustained Remissions in Leukemia. $N$ Engl J Med. 371: 1507-1517, 2014.

45) Porter, D.L., et al.: Chimeric antigen receptor $\mathrm{T}$ cells persist and induce sustained remissions in relapsed refractory chronic lymphocytic leukemia. Sci Transl Med. 7: 303ra139, 2015.

46) Lee, D.W., et al.: T cells expressing CD19 chimeric antigen receptors for acute lymphoblastic leukaemia in children and young adults: a phase 1 dose-escalation trial. Lancet. 385: 517-528, 2015.

47) Louis, C.U., et al.: Antitumor activity and longterm fate of chimeric antigen receptor-positive $\mathrm{T}$ cells in patients with neuroblastoma. Blood. 118: 6050-6056, 2011.

48) Park, J.R., et al.: Adoptive transfer of chimeric antigen receptor re-directed cytolytic $\mathrm{T}$ lymphocyte clones in patients with neuroblastoma. Mol Ther. 15: 825-833, 2007.

49) Morgan, R.A., et al.: Case report of a serious adverse event following the administration of $\mathrm{T}$ cells transduced with a chimeric antigen receptor recognizing ERBB2. Mol Ther. 18: 843-851, 2010.

50) Ahmed, N., et al.: Human Epidermal Growth Factor Receptor 2 (HER2) -Specific Chimeric Antigen Receptor-Modified T Cells for the Immunotherapy of HER2-Positive Sarcoma. J Clin Oncol. 33: 1688-1696, 2015.

51) Parkhurst, M.R., et al.: T cells targeting carcinoembryonic antigen can mediate regression of metastatic colorectal cancer but induce severe transient colitis. Mol Ther. 19: 620-626, 2011.

52) Beatty, G.L., et al.: Mesothelin-specific chimeric antigen receptor mRNA-engineered $\mathrm{T}$ cells induce anti-tumor activity in solid malignancies. Cancer Immunol Res. 2: 112-120, 2014.

53) Yaguchi, T., et al.: The mechanisms of cancer immunoescape and development of overcoming strategies. Int J Hematol. 93: 294-300, 2011.

54) Yaguchi, T., Kawakami, Y.: Cancer-induced heterogeneous immunosuppressive tumor microen- vironments and their personalized modulation. Int Immunol. 28: 393-399, 2016.

55) Spranger, S., et al.: Melanoma-intrinsic $\beta$-catenin signalling prevents anti-tumour immunity. Nature. 523: 231-235, 2015.

56) Yaguchi, T., et al.: Immune suppression and resistance mediated by constitutive activation of Wnt/ $\beta$-catenin signaling in human melanoma cells. $J$ Immunol. 189: 2110-2117, 2012.

57) Sumimoto, H., et al.: The BRAF-MAPK signaling pathway is essential for cancer-immune evasion in human melanoma cells. J Exp Med. 203: 16511656, 2006.

58) Sumimoto, H., et al.: Inhibition of growth and invasive ability of melanoma by inactivation of mutated BRAF with lentivirus-mediated RNA interference. Oncogene. 23: 6031-6039, 2004.

59) Iwata-Kajihara, T., et al.: Enhanced cancer immunotherapy using STAT3-depleted dendritic cells with high Th1-inducing ability and resistance to cancer cell-derived inhibitory factors. J Immunol. 187: 27-36, 2011.

60) Nishio, H., et al.: Immunosuppression through constitutively activated NF- $\kappa \mathrm{B}$ signalling in human ovarian cancer and its reversal by an NF- $\mathrm{BB}$ inhibitor. Br J Cancer. 110: 2965-2974, 2014.

61) Ko, J.S., et al.: Sunitinib mediates reversal of myeloid-derived suppressor cell accumulation in renal cell carcinoma patients. Clin Cancer Res. 15: 2148-2157, 2009.

62) Koya, R.C., et al.: BRAF inhibitor vemurafenib improves the antitumor activity of adoptive cell immunotherapy. Cancer Res. 72: 3928-3937, 2012.

63) Matsuzaki, J., et al.: Tumor-infiltrating NY-ESO-1specific $\mathrm{CD} 8+\mathrm{T}$ cells are negatively regulated by LAG-3 and PD-1 in human ovarian cancer. Proc Natl Acad Sci U S A. 107: 7875-7880, 2010.

64) Fourcade, J., et al.: Upregulation of Tim-3 and PD-1 expression is associated with tumor antigenspecific CD8+ $\mathrm{T}$ cell dysfunction in melanoma patients. $J$ Exp Med. 207: 2175-2186, 2010.

65) Inozume, T., et al.: Melanoma Cells Control Antimelanoma CTL Responses via Interaction between TIGIT and CD155 in the Effector Phase. $J$ Invest Dermatol. 136: 255-263, 2016.

66) Sampson, J.H., et al.: EGFRvIII mCAR-modified T-cell therapy cures mice with established intracerebral glioma and generates host immunity against tumor-antigen loss. Clin Cancer Res. 20: 972984, 2014 
67) Fridman, W.H., et al.: The immune contexture in human tumours: impact on clinical outcome. Nat Rev Cancer. 12: 298-306, 2012.

68) Mattes, J., et al.: Immunotherapy of cytotoxic $T$ cell-resistant tumors by T helper 2 cells: an eotaxin and STAT6-dependent process. J Exp Med. 197: 387-393, 2003.

69) Nishimura, T., et al.: Distinct role of antigenspecific T helper type 1 (Th1) and Th2 cells in tumor eradication in vivo. $J$ Exp Med. 190: 617627, 1999.

70) Kim, H.-J., Cantor, H.: CD4 T-cell subsets and tumor immunity: the helpful and the not-so-helpful. Cancer Immunol Res. 2: 91-98, 2014.

71) Corthay, A., et al.: Primary antitumor immune response mediated by CD4+ T cells. Immunity. 22: 371-383, 2005.

72) Nishimura, T., et al.: The critical role of Th1dominant immunity in tumor Immunology. Cancer Chemother Pharmacol. 46: S52-61, 2000.

73) Tran, E., et al.: Cancer immunotherapy based on mutation-specific CD4+ T cells in a patient with epithelial cancer. Science. 344: 641-645, 2014.

74) Hunder, N.N., et al.: Treatment of metastatic melanoma with autologous CD4+ T cells against NYESO-1. N Engl J Med. 358: 2698-2703, 2008.
75) Bettelli, E., et al.: Reciprocal developmental pathways for the generation of pathogenic effector TH17 and regulatory T cells. Nature. 441: 235-238, 2006.

76) Zou, W., Restifo, N.P.: $\mathrm{T}(\mathrm{H}) 17$ cells in tumour immunity and immunotherapy. Nat Rev Immunol. 10: 248-256, 2010.

77) Martin-Orozco, N., et al.: T Helper 17 Cells Promote Cytotoxic T Cell Activation in Tumor Immunity. Immunity. 31: 787-798, 2009.

78) Muranski, P., et al.: Tumor-specific Th17-polarized cells eradicate large established melanoma. Blood. 112: 362-373, 2008.

79) Purwar, R., et al.: Robust tumor immunity to melanoma mediated by interleukin-9-producing T cells. Nat Med. 18: 1248-1253, 2012.

80) Lu, Y., et al.: Th9 cells promote antitumor immune responses in vivo. J Clin Invest. 122: 4160-4171, 2012.

81) Li, Y., et al.: IL-21 influences the frequency, phenotype, and affinity of the antigen-specific CD8 T cell response. J Immunol. 175: 2261-2269, 2005.

82) Kawakami, Y., et al.: Development of personalized combination cancer immunotherapy based on the patients' immune status. Tsukasa Seya., et al. eds. Inflamamtion and immunity in cancer, Springer, pp255-266, 2015. 